University of South Carolina

Scholar Commons

$12-1997$

\title{
A New Dynamic Mechanism of Topological Charge Creation in a Commensurate One-Dimensional Charge Density Wave Near the Contact with a Normal Metal
}

\author{
A. S. Rozhavsky \\ Yuriy V. Pershin Dr \\ University of South Carolina - Columbia, pershin@physics.sc.edu
}

A. S. Kovalev

Follow this and additional works at: https://scholarcommons.sc.edu/phys_facpub

Part of the Physics Commons

\section{Publication Info}

Published in Low Temperature Physics, Volume 23, Issue 12, 1997, pages 996-999.

Rozhavsky, A. S., Pershin, Y. V., \& Kovalev, A. S. (1997). A new dynamic mechanism of topological charge creation in a commensurate one-dimensional charge density wave near the contact with a normal metal. Low Temperature Physics, 23(12), 996-999.

(c) Low Temperature Physics, 1997, American Institute of Physics

http://ltp.aip.org/resource/1/ltpheg/v23/i12/p996_s1?isAuthorized=no

This Article is brought to you by the Physics and Astronomy, Department of at Scholar Commons. It has been accepted for inclusion in Faculty Publications by an authorized administrator of Scholar Commons. For more information, please contact digres@mailbox.sc.edu. 


\title{
A new dynamic mechanism of topological charge creation in a commensurate one- dimensional charge density wave near the contact with a normal metal
}

\author{
A. S. Rozhavsky, Yu. V. Pershin, and A. S. Kovalev
}

B. Verkin Institute for Low Temperature Physics and Engineering, National Academy of Sciences of the Ukraine, 310164 Kharkov, Ukraine*

(Submitted July 11, 1997; revised August 3, 1997)

Fiz. Nizk. Temp. 23, 1325-1330 (December 1997)

The dynamics of conversion of conduction electrons into topological solitons of a charge density wave (CDW) commensurability in an external electric field is investigated. A novel mechanism of initiation of a nonlinear CDW current in the vicinity of the interface between a CDW and a normal metal is revealed and explained. The nonlinear current is produced by the conduction solitons created by the moving profile of the order parameter, formed during the conversion of electrons into collective phase excitations of a CDW. The field dependence of the current is of threshold nature and has no analogs in the bulk mechanisms of CDW nonlinearity. (C) 1997 American Institute of Physics. [S1063-777X(97)01012-8]

\section{INTRODUCTION}

It is well known that several quasi-one-dimensional metals are transformed into the Peierls-Fröhlich phase as a result of cooling, which is accompanied by the emergence of a lattice superstructure along the unidimensionality direction with a period $\pi / k_{F}$. The static lattice deformation modulus creates a gap $2 \Delta$ in the one-electron spectrum, while the phase of the order parameter $\Delta \exp (i \varphi)$ is electrically active and causes a collective response of a Peierls insulator (PI) to an external field. The deformations of the lattice are called charge density waves (CDW).

Study of quasi-one-dimensional conductors with CDW is interesting primarily because they exhibit nonlinear electrical conductivity in very weak electric fields (see, for example, the reviews in Refs. 1 and 2). The nonlinear conductivity is of the threshold type and has always been attributed to processes occurring in the bulk of the conductor. There are two theoretical approaches to this phenomenon, viz., the classical approach and the quantum approach. ${ }^{1,2}$ In both approaches, the charge transport in a CDW is presented as a collective mode, i.e., the Peierls-Fröhlich order parameter phase, when the charge fluctuations $\delta \rho$ and the CDW current $j$ are described by Fröhlich relations for a single chain (see, for example, Ref. 1):

$$
\delta \rho=\frac{e}{\pi} \frac{\partial \varphi}{\partial x}, \quad j=-\frac{e}{\pi} \frac{\partial \varphi}{\partial t},
$$

where $x$ is the direction of unidimensionality.

The CDW on a single chain is always described by the sine-Gordon (SG) equation. The SG solitons play the role of charge carriers.

In the classical model, the nonlinearity of electrical conductivity has the form ${ }^{1}$

$$
j \sim \sqrt{E^{2}-E_{T}^{2}}, \quad E>E_{T} .
$$

The threshold field $E_{T}$ is connected with the bulk term in the SG equation.

The quantum model connects the nonlinearity with the tunnel creation of soliton-antisoliton $(s \bar{s})$ pairs by an electric field, and the current is defined as ${ }^{2}$

$$
j \sim\left(E-E_{T}\right) \exp \left(-\frac{E_{0}}{E-E_{T}}\right), \quad E>E_{T} .
$$

The threshold field $E_{T}$ is determined by the Coulomb interaction between a soliton and an antisoliton.

However, the modern state of the experiment does not permit us to give preference to any of the models, hence both approaches are equivalent at present, and probably complement each other.

It has become clear recently that an important role in the conductivity of CDW is played by contact phenomena at the interface between a CDW and a normal metal, where the conduction electron current is transformed into the collective CDW current. Qualitatively, the need for the existence of a conversion channel is dictated by the instability of a free electron or hole in a quasi-one-dimensional Peierls lattice to self-trapping with the formation of a collective charge. ${ }^{3,4}$ This channel causes a finite conduction through the metalCDW interface at temperatures $T \ll \Delta$, when the conventional one-electron current is exponentially suppressed $[\sim \exp (-\Delta / T)]$ (see, for example, Ref. 5).

It is not obvious beforehand whether an additional nonlinearity emerges in the conversion channel. This problem is studied in the present work where it is shown that a nonlinear conductivity emerges in a simplified one-dimensional model. This conductivity has a structure different from Eqs. (1) and (2):

$$
j \sim \frac{\sqrt{E-E_{T}} E}{A+\sqrt{E-E_{T}}},
$$

where $E_{T}$ is defined by the bulk term in the sine-Gordon equation like in the classical model.

Investigations of the dynamics of the conversion process were started in Refs. 6 and 7. An instanton mechanism was proposed in Ref. 6 for the transition of free charges from the chemical potential level at the metal-CDW interface to the PI condensate (valence band) with the formation of a collective charge (CDW phase profile) localized at distances of the order of $\xi_{0}=\hbar V_{F} / \Delta$ from the interface. Transition to the condensate is always made by two electrons, which is reminis- 
cent of Andreev scattering from the point of view of the outer boundary relative to the PI. Unlike the case of superconductors in which $\Delta$ is the only parameter determining the space and time dynamics of the order parameter, three welldefined time scales exist in a $\mathrm{PI}^{2,3,6,7}$ :

(1) the time of formation of the quasiparticle spectrum $\tau_{S} \sim \hbar / \Delta$

(2) the time of deformation of the order parameter modulus $\tau_{\Delta} \sim \bar{\omega}^{-1}$, where $\bar{\omega}$ is of the order of Debye frequency $\bar{\omega} \ll \Delta$

(3) the phase evolution time of the order parameter $\tau_{\varphi} \gg \tau_{\Delta} \gg \tau_{s}$.

These parameters make it possible to formulate and consistently solve the problem of transformation of electron charge through the interface. A quasiparticle level is formed on gap fluctuation (instanton) over a time $\tau_{s}$. The fluctuation itself takes place over a time $\tau_{\Delta}$, the bound level is absorbed by the valence band, and the initial condition $\delta \varphi=\varphi(x=\infty)-\varphi(x=0)=2 \pi$ is formed for the problem on the evolution of the phase from the boundary $x=0$ towards $x>0$. This condition corresponds to a charge $2 e$ [see Eq. (1)]. A steadily moving CDW profile is obtained from the initial condition in time $\tau_{\varphi}{ }^{7}$ ?

Earlier, we studied ${ }^{7}$ the evolution of the initial condition in a cluster of the nearest chains carrying a noncommensurate $\mathrm{CDW}$. The role of the bulk term in the sine-Gordon equation was played by interaction of the type $\sin \left(\varphi_{i}-\varphi_{i+1}\right)$ between chains. In the equation for a commensurate $\mathrm{CDW}$, each chain acquires an additional term $\sin \left(M \varphi_{i}\right)$, where $M$ is an integer. ${ }^{1,2}$ Accordingly, a topological soliton has an asymptotic form $\delta \varphi=\varphi(x=\infty)-\varphi(x=-\infty)=2 \pi / M$. Such a soliton is a stable carrier of the fractional charge $2 e / M$ in a CDW. ${ }^{2}$ It is certainly interesting to study the evolution of the initial condition $\delta \varphi=2 \pi$ in $M$ solitons and to study their dynamics in an electric field. Such a problem is formulated and solved in the present work for a single chain.

In Ref. 7, we reduced the Cauchy problem for a CDW to an exactly integrable problem and solved it by the inverse scattering problem method. In an external electric field, the exact integrability is violated on the right-hand side of the sine-Gordon equation, and the equation is solved numerically. It was found that $s \bar{s}$ pairs are created in an electric field with an intensity inversely proportional to time $\bar{\tau}$ (14), which determines the current (4).

We believe that the nonlinear conductivity (4) can be explained as follows: the initial CDW profile at the interface has an energy of the order $\Delta$ which is considerably higher than static energy of a topological soliton carrying a charge $2 e / M$. Away from the interface, nonlinear deformations are created in the moving front region from the high-energy initial profile in a time $\tau_{0}$. During a time of the order of $\sqrt{2 d /\left(E-E_{T}\right)}$ ( $d$ is the soliton width), these deformations are transformed into topological solitons with an asymptotic form $\delta \varphi=2 \pi / M$, or antisolitons with $\delta \varphi=-2 \pi / M$ (depending on the direction of the electric field) moving behind the front along or against the field. Accordingly, the total time for the creation of a stable topological charge $\pm 2 e / M$ is $\bar{\tau}=\tau_{0}+\sqrt{2 d /\left(E-E_{T}\right)}$. The creation frequency $\bar{\tau}^{-1}$ determines the number of charges created, i.e., the nonlinear conductivity (4).

A similar dynamic effect of multiple creation of domain walls for a moving domain boundary was studied in Ref. 8 .

\section{MODEL AND NUMERICAL ANALYSIS}

Suppose that a CDW occupies a semi-axis $x \geqslant 0$. The Lagrangian of the CDW in a field is defined as (see, for example, Ref. 2)

$$
\begin{aligned}
L= & \frac{1}{\pi \hbar V_{F}}\left\{\frac{\Delta^{2}}{\bar{\omega}^{2}}\left(\frac{\partial \varphi}{\partial t}\right)^{2}-\frac{\hbar^{2} V_{F}^{2}}{4}\left(\frac{\partial \varphi}{\partial x}\right)^{2}\right. \\
& \left.+\frac{\Delta^{2}}{\bar{\omega}^{2}} \frac{2}{M^{2}} \omega_{0}^{2} \cos M \varphi+\frac{e}{\pi} \hbar V_{F} E\left(\varphi-\varphi_{0}\right)\right\},
\end{aligned}
$$

where $\omega_{0}$ is the commensurability frequency. Here, $\varphi_{0}$ is the phase for $x \rightarrow \pm \infty$. Such a form of notation for the last term in Eq. (5) takes into account the fact that the electric field acts on CDW only in the region where $|\partial \varphi / \partial x| \neq 0$.

In a nonzero field $E$, the quantity $\varphi_{0}$ is renormalized as seen from the equation of motion, which can be represented in dimensionless units

$$
\tau=\omega_{0} t \sqrt{2 / M}, \quad y=x\left(\frac{\hbar V_{F} \bar{\omega}}{2 \Delta \omega_{0}} \sqrt{M / 2}\right),
$$

for the variable $\chi=\varphi-\varphi_{0}$.

The equation of motion has the form

$$
\frac{\partial^{2} \chi}{\partial \tau^{2}}-\frac{\partial^{2} \chi}{\partial y^{2}}+\sin M\left(\chi+\varphi_{0}\right)=\varepsilon,
$$

where

$$
\varepsilon=\frac{e}{2 \pi} \hbar V_{F} \frac{M \bar{\omega}^{2}}{\Delta^{2} \omega_{0}^{2}} E
$$

and $\varphi_{0}=\arcsin \varepsilon$

Equation (7) is supplemented by the initial and boundary conditions corresponding to the above hierarchy of time in the conversion problem:

$$
\begin{aligned}
& \left.\frac{\partial \chi}{\partial \tau}\right|_{r=0}=0, \\
& \chi(\tau=0)=-2 \pi \theta\left(\bar{\xi}_{0}-y\right)-\varphi_{0}^{\prime}(\varepsilon), \\
& \chi(y=0)=-2 \pi-\varphi_{0}(\varepsilon),
\end{aligned}
$$

where $\bar{\xi}_{0}$ is the coherence length $\xi_{0}=\hbar V_{F} / \Delta$ in dimensionless variables (6), $\bar{\xi}_{0} \ll 1$, and $\theta(y)$ is the Heaviside function.

Note that the small scale of variation of the initial condition $\bar{\xi}_{0} \ll 1$ cannot emerge formally in the solution of a purely phase problem with Lagrangian (5) since the latter corresponds to the long-wave approximation $\left|\varphi^{\prime}\right| \ll \xi_{0}^{-1}{ }^{2}$ It was mentioned above (see Ref. 7 also) that such an initial condition is the result of joining of the instanton solution ${ }^{6}$ formed during the time $\tau_{\Delta}$ with a long-wave description valid for $\tau \geqslant \tau_{\varphi}$. 
t1
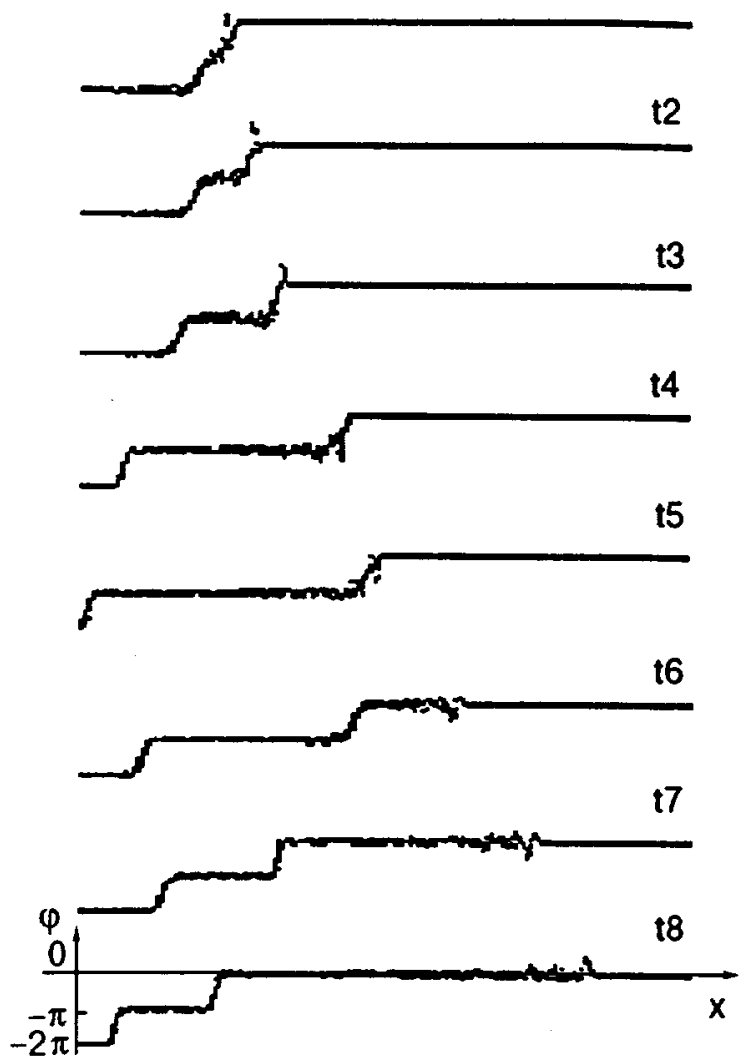

FIG. 1. Evolution of the initial condition in a retarding field $\varepsilon=-0.05$, $M=2: t 1<t 2<t 3<t 4<t 5<t 6<t 7<t 8$.

Equation (7) with the boundary conditions (9) was solved numerically by the method of finite differences. The difference equation corresponding to Eq. (7) has the form

$$
\begin{gathered}
\frac{\chi_{i+1, k}+\chi_{i-1, k}-2 \chi_{i, k}}{(\Delta \tau)^{2}}-\frac{\chi_{i, k+1}+\chi_{i, k-1}-2 \chi_{i, k}}{(\Delta y)^{2}} \\
+\sin M\left(\chi+\varphi_{0}\right)=\varepsilon,
\end{gathered}
$$

where $\Delta \tau$ is the time step, $\Delta y$ is the coordinate step, and $\chi_{i, k}=\chi(\Delta \tau i, \Delta y k)$. Equation (10) was solved for different values of $\Delta \tau, \Delta y$, and $M$, and under different initial conditions (9). Typical solutions have been constructed in Figs. 1, 2 , and 3 . The number of sites $(k)$ is 10000 . It was found that a change in the initial conditions (9) has no significant effect on the solution of Eq. (7).

In a weakly retarding field $|\varepsilon| \ll 0.1$ (Fig. 1), the initial profile moving against the field loses stability after some time and simply splits into commensurability solitons. The latter are retarded, reverse their direction, and eventually gather near $x=0$. Radiation propagating with the maximum speed is observed. With increasing field, charge creation by the field is observed additionally (Fig. 2).

In an accelerating field, the initial condition trivially decomposes into topological solitons with time for $\varepsilon<0.1$, while the field merely affects the time of formation of solitons and their velocity. Movement of radiation at the highest velocity was observed in front of the profile. For $\varepsilon>0.1$, charge creation by the field is observed (Fig. 3). A similar

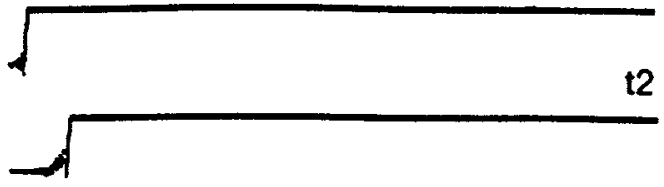

t3

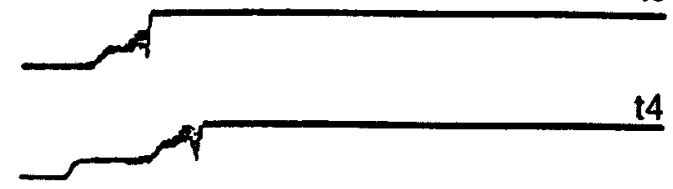

t5
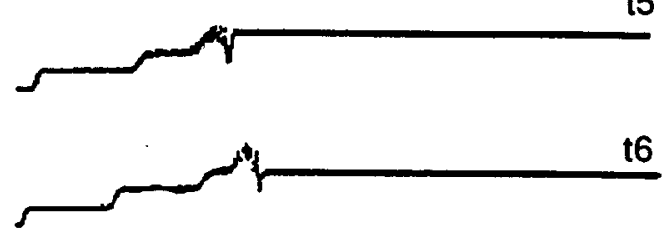

t7

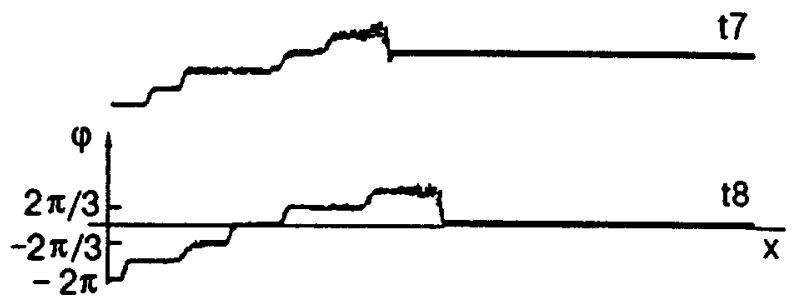

FIG. 2. Evolution of the initial condition in a retarding field $\varepsilon=-0.3$, $M=3$. CDW solitons with an asymptotic form $2 \pi / 3$ are produced from the initial condition $(t 1)$ with increasing time: $t 1<t 2<t 3<t 4<t 5<t 6<t 7<t 8$.

effect was also observed in Ref. 9 during a numerical analysis of the motion of Josephson vortices in a system with dissipation. However, the difference lies in that, instead of an infinite number, we are looking at a finite number of created charges, this number depending on the field (Fig. 4). The dependence of the charge creation time on the field is shown in Fig. 5 and is approximated well by the law $\bar{\tau}=A+B / \sqrt{\varepsilon-\varepsilon_{T}}$.

A reversal of the sign of created charges upon a change in the direction of the field unambiguously indicates that the mechanism of their formation is polarization: for $\varepsilon>0$, the soliton moves along the field, and the antisoliton moves against the field; for $\varepsilon<0, s$ and $\bar{s}$ interchange their places.

The time of formation of free charge carriers is estimated as follows.

Suppose that, in a CDW profile moving with a practically limiting velocity, nonlinear fluctuations produce a deformation of the front with $\delta \varphi=0$ over a time $\tau_{0}$. In the absence of a field, such a process does not lead to charge creation since such a deformation moves with the front and is not polarized.

We shall assume that, in the presence of a field $\varepsilon>0$, the leading front of the created deformation continues to move with the CDW front with a limiting velocity, while the trailing front gets retarded by the field and is ultimately transformed into a soliton with $\delta \varphi_{S}=2 \pi / M$, which subsequently reverses its direction. The equations of motion for the lead- 


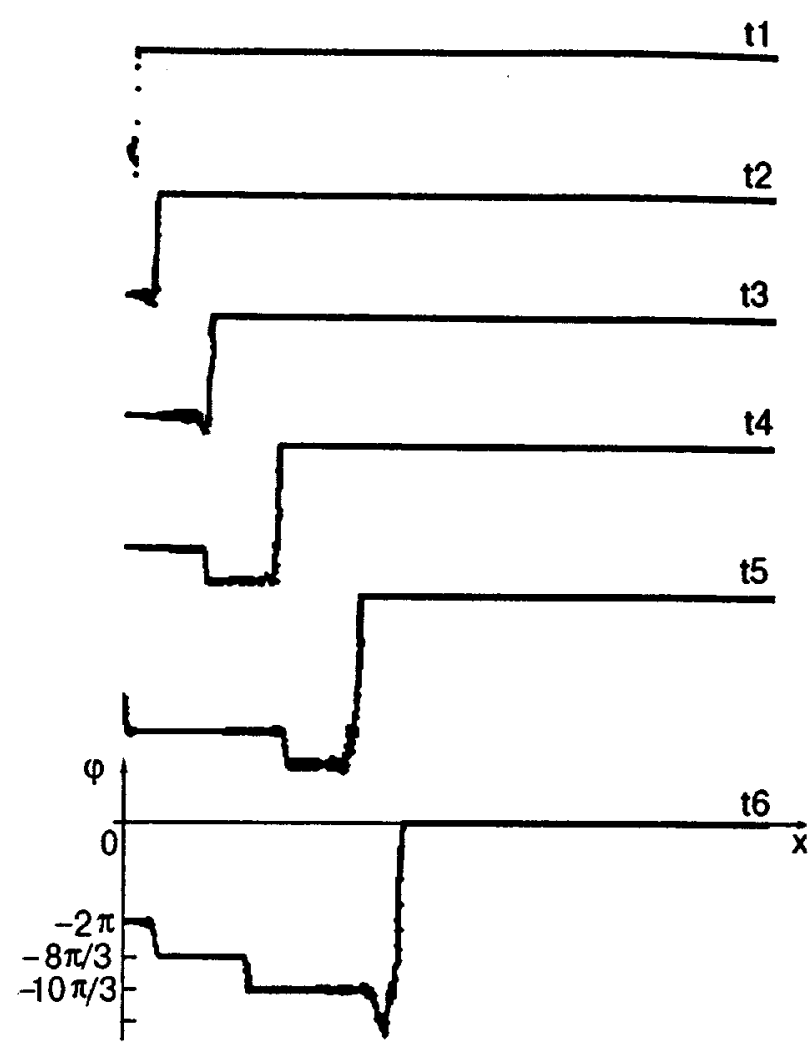

FIG. 3. Evolution of the initial condition in an accelerating field $\varepsilon=0.3$, $M=3$. CDW solitons with an asymptotic form $2 \pi / 3$ are produced from the initial condition $(t 1)$ with increasing time. $t 1<t 2<t 3<t 4<t 5<t 6$.

ing front $x_{+}$and the trailing front $x_{-}$of deformation have the form

$$
\begin{aligned}
& (+): x_{+}=C_{0}\left(\tau-\tau_{0}\right)+x_{0}, \\
& (-): x_{-}=\frac{\left(\varepsilon-\varepsilon_{T}\right) \tau^{2}}{2}+\lambda \tau+C,
\end{aligned}
$$

where $C_{0}$ is the maximum velocity in the system, $\varepsilon_{T}$ is the threshold field, while the constants $\lambda$ and $C$ are determined from the conditions

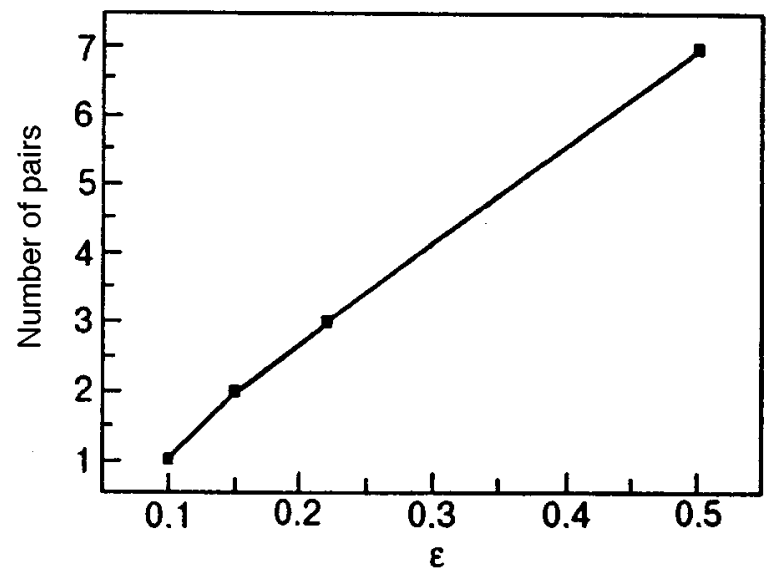

FIG. 4. Field dependence of the number of topological solitons created, $\varepsilon>0, M=3$.

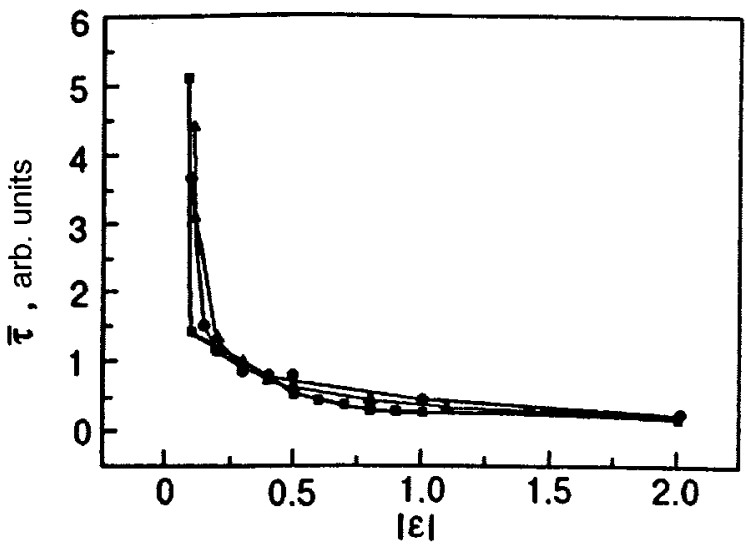

FIG. 5. Dependence of the topological charge creation time on field $|\varepsilon|$. Numerical method for $\varepsilon>0(\boldsymbol{\square})$; numerical method for $\varepsilon<0(\mathbf{\bullet})$, results obtained by using formula (14) (dark triangles).

$$
x_{+}\left(\tau_{0}\right)=x_{0},\left.\quad \frac{\partial x_{-}}{\partial \tau}\right|_{\tau=\tau_{0}}=C_{0} .
$$

As soon as the field $\varepsilon-\varepsilon_{T}$ separates the centers $x_{+}$and $x_{-}$by a distance of the order of the kink width $d$ over a time $\bar{\tau}$, we can see that the trailing profile of the created deformation moves like a free charge, i.e.,

$$
d=x_{+}-x_{-}=\left\{-\frac{\bar{\tau}^{2}}{2}+\tau_{0} \bar{\tau}-\frac{\tau_{0}^{2}}{2}\right\}\left(\varepsilon-\varepsilon_{T}\right) .
$$

From the last equation, we obtain

$$
\bar{\tau}=\tau_{0}+\sqrt{2 d /\left(\varepsilon-\varepsilon_{T}\right)},
$$

as is indeed observed in the numerical experiment (Fig. 5). The quantity $\bar{\tau}^{-1}(\varepsilon)$ defines the intensity of free charge creation, i.e., the current (4).

Thus, we have discovered and explained a new mechanism of formation of the nonlinear CDW current near the interface with a normal conductor. This mechanism supplements the known mechanisms studied for an infinite CDW.

*E-mail: rozhavsky@ilt.kharkov.ua

${ }^{1}$ P. Manceau (Ed.), Electronic Properties of Inorganic Quasi-OneDimensional Compounds, Riedel Co., Dordrecht (1985), parts 1 and 2; G. Grüner (Ed.), Density Waves in Solids, Addison-Wesley, Reading, Mass. (1994).

${ }^{2}$ I. V. Krive, A. S. Rozhavsky, and I. O. Kulik, Fiz. Nizk. Temp. 12, 635 (1986) [Sov. J. Low Temp. Phys. 12, 360 (1986)].

${ }^{3}$ S. A. Brazovskii, Zh. Éksp. Teor. Fiz. 51, 342 (1980) [sic].

${ }^{4}$ S. Brazovskii and S. Matveenko. J. Plasma Phys. 2, 725 (1992).

${ }^{5}$ B. Rejaei and G. E. Bauer, Phys. Rev. B 54, 8487 (1996).

${ }^{6}$ I. V. Krive, A. S. Rozhavsky, and V. A. Rubakov, Pis'ma Zh. Éksp. Teor. Fiz. 46, 99 (1987) [JETP Lett. 46, 121 (1987)].

${ }^{7}$ A. S. Kovalev, Yu. V. Pershin, and A. S. Rozhavsky, Phys. Rev. B 53, 16227 (1996); Fiz. Nizk. Temp. 22, 322 (1996) [ Low Temp. Phys. 22, 253 (1996)].

${ }^{8}$ V. G. Bar'yakhtar, B. I. Ivanov, and M. V. Chetkin, Usp. Fiz. Nauk, 146, 417 (1985) [Sov. Phys. Usp. 28, 563 (1985)].

${ }^{9}$ K. Nakagama, Y. Onodera, T. Nakamura, and R. Sato, J. Appl. Phys. 45, 4095 (1974).

Translated by R. S. Wadhwa 